\title{
Handling stress-induced ruminal microbiota changes reduce grass hay degradability in sheep
}

\author{
Y. Yoshihara ${ }^{1}$ and Y. Ogawa \\ Mie University, 1577 Kurimamachiya-cho, Tsu City, Mie Prefecture, 514-8507, Japan
}

KEY WORDS: animal welfare, cellulolytic bacteria, forage intake, rumen digestibility, sheep, stress

Received: 2 December 2020

Revised: $\quad 9$ March 2021

Accepted: 16 March 2021
${ }^{1}$ Corresponding author:

e-mail: marmota.sibirica@gmail.com

\begin{abstract}
Animal welfare is a critical aspect of farming. Poor practices are strongly linked to deterioration in animal health and performance, as they suppress immune functions and stress. Negative handling can be a strong stressor while positive handling can reduce stress in farm animals. We hypothesized that handling stress would alter rumen environments and consequently microflora, which would reduce the ability of the rumen to digest hay. To test this hypothesis, we exposed four ewes to positive (free-grazing) and negative handling protocols (transportation or combination of novel stimulation and social isolation), as well as examined a control that received no treatment, and then compare the rumen liquid collected from all ewes. The extracted rumen liquid was subjected to in vitro grass hay degradability assay, and genetic approaches were used to identify ruminal microflora. It was shown that feed intake and in vitro grass hay degradability were reduced under the negative handling protocols. Further, sheep exposed to stress had more potentially pathogenic bacteria and fewer cellulolytic bacteria such as Firmicutes, as well as microorganism diversity in the rumen was lower. So, it was concluded that handling stress impairs the ability of the rumen to digest hay due to altered rumen microflora. It is therefore important to recognize potential animal production risks posed by handling stress, which has welfare implications for these farm animals.
\end{abstract}

\section{Introduction}

There is an increasing global demand for animal welfare-friendly farming systems as lack of animal welfare is strongly linked to deterioration in the health of the animal, performance and meat quality through suppressed immune functions and stress (Broom and Fraser, 2015). Inappropriate management by farmers during handling, such as the presentation of novel stimuli, transport, violence and the use of restraints, is a strong stressor for farm animals (Grandin, 2007). Welfare-friendly management such as the provision of environmental enrichment or exercise area under indoor housing can reduce stress and offer certain health benefits to cows (Boissy et al., 2007; Von Keyserlingk et al., 2009; Charlton et al., 2011).
For example, in comparison to positive handling, negative handling increases fear of humans in cattle and results in increased heart rate and total cortisol concentration in the blood (Breuer et al., 2003; Yardimci et al., 2013; Lindahl et al., 2016).

In animals, stressors affect the gastric acid secretion and their function resulted from the activation balance of sympathetic and parasympathetic nervous systems (Murakami et al., 1985; Lenz et al., 1988). Heat stress reduces the amount of saliva produced, which may impair the rumen functionality of cows (Kadzere et al., 2002). Therefore, it is not surprising that handling stress-induced changes in the production of saliva by ruminant will in turn affect the rumen environment and ruminal microflora. Further, this alteration of microflora may be detrimental to the 
digestibility of grass and consequently animal production. However, the sequential flow from stress to grass digestibility via rumen environment and microflora has not yet been fully understood. We, therefore, studied the effect of handling stress on grass hay degradability examined by using a rumen in vitro batch culture technique and diversity of microflora in the rumen liquid examined by a genetic approach.

\section{Material and methods}

\section{Animal management}

The study was conducted at Mie University and Mie Prefectural Livestock Research Institute, located in central Japan. Suffolk ewes aged between 5-10 years that had been kept for one month in individual pens in the same barn were used. All sheep were fed each day $250 \mathrm{~g}$ of feed concentrate (crude protein $13.0 \%$, ether extract $3.1 \%$, nitrogenfree extracts $49.9 \%$, crude fibre $22.7 \%$, crude ash $3.0 \%$ ) and $500 \mathrm{~g}$ of Italian ryegrass hay (crude protein $5.6 \%$, ether extract $1.3 \%$, nitrogen-free extracts $48.9 \%$, crude fibre $29.2 \%$, crude ash $5.6 \%$ ) in the morning (8:00-9:00) and evening (15:00-16:00). Before feeding, leftover forage in the feeder was removed and weighed for calculation of feed intake.

On each day, sheep were exposed to different stress-inducing handling protocols (transportation; control in which no action was taken; free grazing; combination of novel stimulation and social isolation). On the first day, all sheep were transported by truck for a total of $5 \mathrm{~h}$. Rumen liquid was extracted twice (day and 5 days after transportation except one sheep from which rumen liquid was collected 3 and 6 days after shipment because of weak animal condition) by cannula from the sheep rumen within the first week after transportation depending on the condition of the sheep (negative handling, 'NH1', Figure 1).
In the second week, the rumen liquid was extracted twice (days 3 and 6 in the week) from all sheep under no treatment (control). In the third week, all sheep were moved from their pens to an open ground without plants for free movement and rumen liquid was extracted twice (days 3 and 6 in the week) from sheep $2 \mathrm{~h}$ after the movement (positive handling, ' $\mathrm{PH}$ '). In the final week every day, all sheep were moved to another room in which each sheep was kept alone for $2 \mathrm{~h}$ with a human in the room scaring the sheep by making loud noises without making any eye contact with animals. Rumen liquid was then extracted twice (days 3 and 6 in the week) from the sheep $2 \mathrm{~h}$ after the treatment ('NH2').

Collection of rumen liquid was done before feeding in the morning. It was filtered through 2 layers of cheesecloth. The rumen liquid was then prepared by the deoxidized method using bubbling $\mathrm{CO}_{2}$ gas. The $\mathrm{pH}$ of the rumen liquid was also recorded.

\section{In vitro degradability measurement of grass hay}

The degradability of Italian ryegrass hay was evaluated using a rumen in vitro batch culture technique as described by Khan and Chaudhry (2010) with the different sources of rumen fluid (stressed $v s$ non-stressed sheep) used as treatments. The hay was ground to pass through a $1-\mathrm{mm}$ screen, and $500 \mathrm{mg}$ of the sample was accurately weighed into a 30-ml bottle with a butyl rubber stopper and aluminium seal. This was anaerobically incubated at $38^{\circ} \mathrm{C}$ for $48 \mathrm{~h}$ under the in vitro conditions with rumen mixed culture (v/v, 1:4 rumen mixed culture: McDougall's buffer solution $\left(9.8 \mathrm{~g} \mathrm{NaHCO}_{3}\right.$, $9.3 \mathrm{~g} \mathrm{Na}_{2} \mathrm{HPO}_{4} \times 12 \mathrm{H}_{2} \mathrm{O}, 0.57 \mathrm{~g} \mathrm{KCl}, 0.47 \mathrm{~g} \mathrm{NaCl}$, $0.12 \mathrm{~g} \mathrm{MgSO}_{4} \times 7 \mathrm{H}_{2} \mathrm{O}, 0.04 \mathrm{~g} \mathrm{CaCl}_{2}, 1000 \mathrm{ml}$ distilled water); McDougall, 1948). In total, 32 samples (4 handling treatments including $\mathrm{PH}, \mathrm{NH} 1$, $\mathrm{NH} 2$ and control $\times 4$ sheep $\times 2$ times sampling of

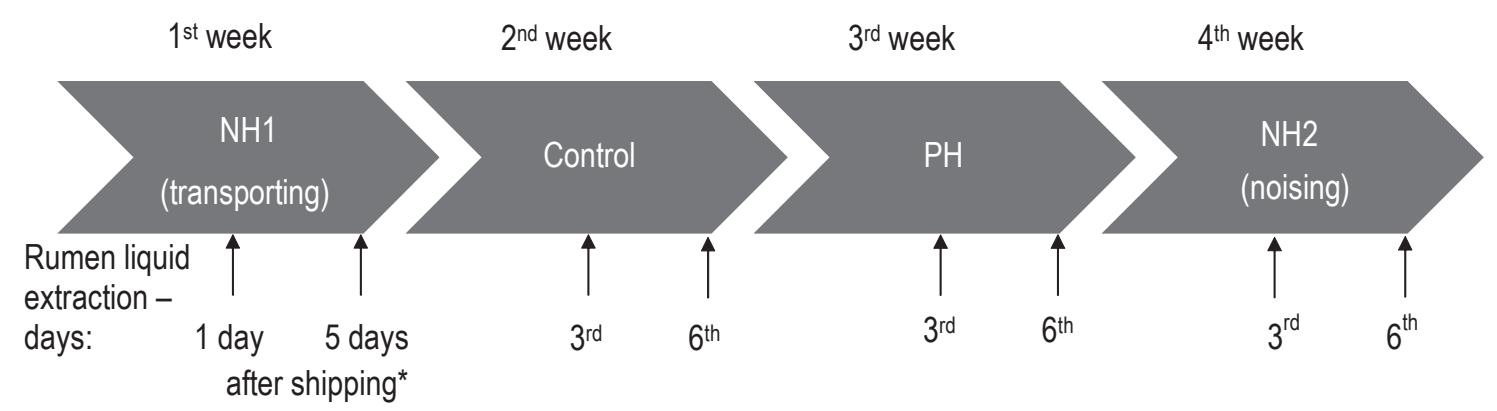

Figure 1. Time-sequence diagram of experimental periods and rumen liquid sampling time

Handling protocols: $\mathrm{NH}$ - negative handling ( $\mathrm{NH} 1$ - transporting; $\mathrm{NH} 2$ - noising); control - without any handling treatment; $\mathrm{PH}$ - positive handling; * - from one sheep rumen liquid was collected 3 and 6 days after the transportation because the sheep looked weak maybe due to the transportation stress 
rumen liquid) were run in three replications and the filtered plant sample was collected on a filter paper (ADVANTEC, Tokyo, Japan), dried and weighed.

\section{Analysis of rumen microorganisms}

The filtered rumen liquid in a separate funnel was left to stand at $39^{\circ} \mathrm{C}$ for $1 \mathrm{~h}$. The upper part of the liquid was separated by centrifuging at $1000 \mathrm{rpm}$ for $5 \mathrm{~min}$, and the lower part of the liquid was separated by centrifuging at $1000 \mathrm{rpm}$ for $5 \mathrm{~min}$ and $12000 \mathrm{rpm}$ for $20 \mathrm{~min}$. The supernatant liquids in each centrifugation including protozoa and bacteria fractions were collected (Sasaki et al., 2000). Separated microorganism fractions underwent a $48 \mathrm{~h}$ freeze-drying process and were weighed.

Genomic DNA of rumen microorganisms was extracted from the samples using a FavorPrep Soil DNA Isolation Mini Kit (Favorgen Biotech Corporation, Ping-Tung, Taiwan) following the manufacturer's instructions. To amplify the DNA of the two groups, PCR primer sets specifically for the V3-4 hypervariable region of the $16 \mathrm{~S}$ rRNA gene for bacteria (e.g., 5'-ACACTCTTTCCCTACACGACGCTCTTCCGATCT-NNNNNCCTACGGGNGGCWGCAG-3') and the 18S rRNA operon for eukaryotes (e.g., ACACTCTTTCCCTACACGACGCTCTTCCGATCTATAACAGGTCTGTGATGCC) were used. The first PCR product was purified by Agencourt AMPure XP (Beckman Coulter, Brea, CA, USA) and used as the template for the second PCR. The second PCR was performed to add complementary sequences for the oligonucleotides that coat the Illumina sequencing flow cell, annealing sites of DNA sequencing primers, and indices to the first PCR products. These PCR steps were conducted independently to add individual indices to each sample using the common forward and indexed reverse primers (Suyama and Matsuki, 2015). The second PCR product was then purified by Agencourt AMPure XP (Beckman Coulter, Brea, CA, USA). The concentrations of each of the second PCR products (libraries) were measured using a Reagent Kit Synergy H1 (Bio Tek, Winooski, VT, USA) and a QuantiFluor dsDNA System (Promega, Madison, WI, USA). The libraries from each sample, each with a different index, were then pooled in equimolar concentrations. Libraries were denatured using fresh $\mathrm{NaOH}(0.2 \mathrm{~N})$ and mixed with $20 \%$ of Illumina-generated PhiX control libraries, as per Illumina's protocols. Approximately $10 \mathrm{pM}$ of the libraries were used for sequencing on an Illumina MiSeq Sequencer using a MiSeq Reagent Kit V3 (Illumina, San Diego, CA, USA).
Raw data obtained was processed and analyzed using the QIIME pipeline software (version 1.8.0; Caporaso et al., 2010). Merged fastq format data was converted to fasta format using fastq to fasta software in the fastx_toolkit package. The sequence reads with an average quality of $<$ Q20 were removed using Sickle software. High-quality pairedend reads were assembled using FLASH software. Chimeric sequences were removed using Usearch V6.1.544 based on the Uchime algorithm (Edgar, 2010) implemented in QIIME. The clean fasta data were aligned into Operational Taxonomic Units (OTUs) at $97 \%$ similarity using open-reference OTU picking against the $16 \mathrm{~S}$ and $18 \mathrm{~S}$ databases. Taxonomic identification was performed at the phylum and genus levels. Shannon's diversity index was calculated at each level.

A general linear model with individuals as a random effect was applied to test statistically significant differences in rumen $\mathrm{pH}$, degradability and the amount of forage intake across stress treatments. Kruskal-Wallis test was applied to compare the compositional change of rumen microorganism among the treatments (control $(\mathrm{n}=8), \mathrm{PH}(\mathrm{n}=8)$, $\mathrm{NH}(\mathrm{n}=16$; NH1 + NH2)). Tukey's post-hoc test and Steel-Dwass's post-hoc test were performed to ascertain which treatments varied significantly from each other. These analyses were performed with Statistica 12 software (Dell Inc., Round Rock, TX, USA). All differences among comparisons with $P \leq$ 0.05 were considered significant.

\section{Results and discussion}

The daily forage intake of sheep was reduced under the negative handling protocols (Figure 2, $P<0.001, \mathrm{~F}=17.1, \mathrm{df}=2$ ). In vitro grass hay degradability was the greatest under the $\mathrm{PH}$ and control conditions $(P<0.001, \mathrm{~F}=9.50, \mathrm{df}=2)$. Ruminal $\mathrm{pH}$ $(P=0.63, \mathrm{~F}=0.49, \mathrm{df}=2)$ and weight of rumen microorganisms $(P=0.09, \mathrm{~F}=3.22, \mathrm{df}=2)$ did not differ significantly among the three handling protocols.

The average number of OTUs per PH, control and $\mathrm{NH}$ treatment detected by the analysis reached 4098, 46037 and 44840 for bacteria and 10756, 16876 and 16740 for eukaryotes, respectively. Diversity index at OTUs level per $\mathrm{PH}$, control and $\mathrm{NH}$ treatment were $3.381,3.373$ and 3.293 for bacteria and $1.423,1.483$ and 1.289 for eukaryotes, respectively. Changes in phyla as a percentage of total sequences in bacterial and eukaryote communities are shown in Table 1. Bacteroidetes and Firmicutes were 

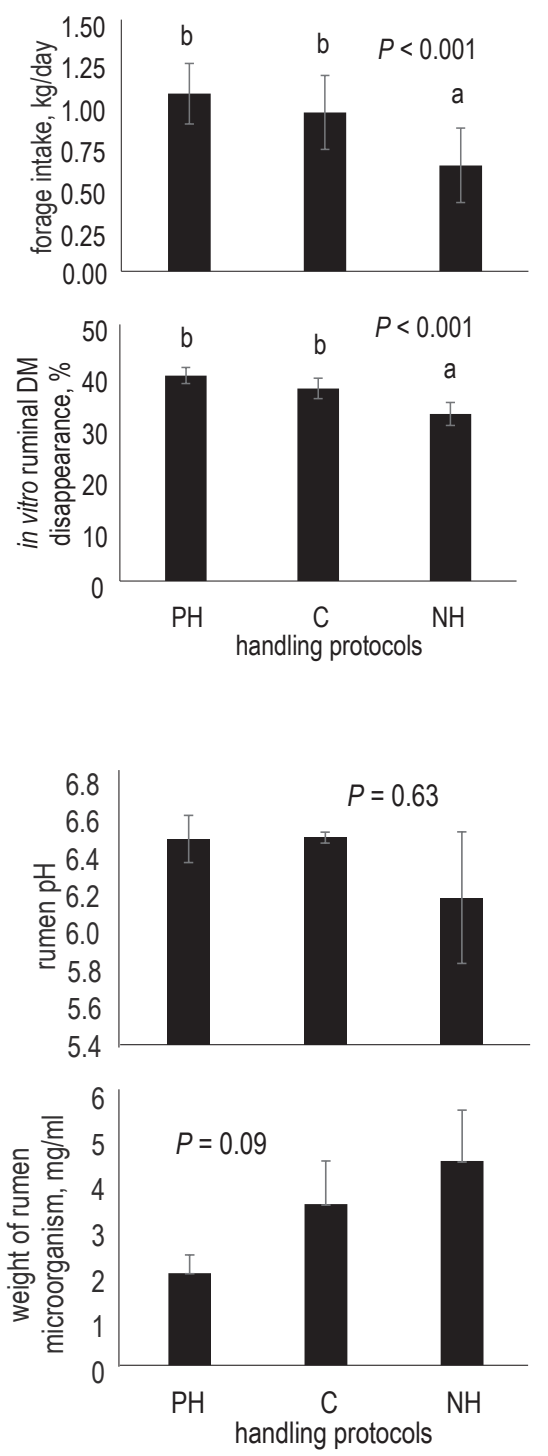

Figure 2. Forage intake, grass hay degradability, rumen $\mathrm{pH}$ and the weight of ruminal microorganisms (mean \pm standard error) for each handling protocol of sheep

Bars with different letters are significantly different according to Tukey's post-hoc test. Handling protocols: $\mathrm{NH}-$ negative handling $(n=16 ; \mathrm{NH} 1+N H 2) ;$ control - without any handling treatment $(n=8)$; $\mathrm{PH}-$ positive handling $(n=8)$

the dominant bacterial phyla in the rumen, regardless of handling treatments. However, Proteobacteria was also a dominant phylum in the stressed sheep.

Proteobacteria include a wide variety of pathogens. Most of the Proteobacteria found from the stressed sheep were Acinetobacter. This species is an opportunistic pathogen and is also found in the rumen of Korean cattle (Chang et al., 2015). In contrast, Firmicutes include important cellulolytic bacterium in rumen such as Ruminococcus spp. (Russell et al., 2009). Ruminococcaceae constituted $10.7,11.1$ and $6.7 \%$ in the rumen bacteria in $\mathrm{PH}$, control and $\mathrm{NH}$ conditions, respectively. So it may be concluded that stressor exposure leads to an over-
Table 1. Effect of handling protocols on changes in phyla as a percentage of total sequences in the rumen bacterial and Eukaryote community

\begin{tabular}{|c|c|c|c|c|}
\hline \multirow{2}{*}{ Phylum } & \multicolumn{3}{|c|}{ Handling protocol } & \multirow{2}{*}{$P$-value } \\
\hline & $\overline{\mathrm{PH}}$ & Control & $\mathrm{NH}$ & \\
\hline \multicolumn{5}{|l|}{ Bacteria } \\
\hline Bacteroidetes & 51.0 & 46.7 & 42.9 & NS \\
\hline Firmicutes & $35.7^{b}$ & $40.0^{\mathrm{b}}$ & $22.9^{\mathrm{a}}$ & $<0.05$ \\
\hline Euryarchaeota (Archaea) & 2.70 & 4.30 & 1.90 & NS \\
\hline TM7 & 4.10 & 2.20 & 2.30 & NS \\
\hline Verrucomicrobia & 1.20 & 1.50 & 1.40 & NS \\
\hline Actinobacteria & 0.60 & 0.90 & 0.20 & NS \\
\hline Tenericutes & 1.00 & 0.80 & 0.30 & NS \\
\hline Proteobacteria & $0.60^{\mathrm{a}}$ & $0.60^{\mathrm{a}}$ & $25.5^{\mathrm{b}}$ & $<0.01$ \\
\hline Lentisphaerae & 0.60 & 0.50 & 0.40 & NS \\
\hline Chloroflexi & 0.20 & 0.30 & 0.20 & NS \\
\hline Cyanobacteria & 0.50 & 0.30 & 0.30 & NS \\
\hline Planctomycetes & 0.20 & 0.30 & 0.10 & NS \\
\hline Spirochaetes & 0.10 & 0.10 & 0.00 & NS \\
\hline WPS-2 & 0.10 & 0.10 & 0.00 & NS \\
\hline Acidobacteria & 0.00 & 0.00 & 0.00 & NS \\
\hline Armatimonadetes & 0.00 & 0.00 & 0.00 & NS \\
\hline Elusimicrobia & 0.10 & 0.00 & 0.00 & NS \\
\hline Fibrobacteres & 0.00 & 0.00 & 0.00 & NS \\
\hline Fusobacteria & 0.00 & 0.00 & 0.00 & NS \\
\hline GN02 & 0.00 & 0.00 & 0.00 & NS \\
\hline LD1 & 0.00 & 0.00 & 0.10 & NS \\
\hline SR1 & 0.10 & 0.00 & 0.10 & NS \\
\hline Synergistetes & 0.10 & 0.00 & 0.00 & NS \\
\hline WS6 & 0.00 & 0.00 & 0.00 & NS \\
\hline Unassigned and others & 1.10 & 1.20 & 1.30 & NS \\
\hline \multicolumn{5}{|l|}{ Eukaryote } \\
\hline Ciliophora & $0.11^{\mathrm{a}}$ & $0.55^{b}$ & $0.37^{b}$ & $<0.01$ \\
\hline Apicomplexa & $0.18^{b}$ & $0.09^{a b}$ & $0.02^{\mathrm{a}}$ & $<0.01$ \\
\hline Fungi & 0.07 & 0.04 & 0.02 & NS \\
\hline Charophyta & 0.01 & 0.01 & 0.03 & NS \\
\hline Parabasalia & 0.01 & 0.01 & 0.03 & NS \\
\hline Metazoa (Animalia) & $0.03^{a}$ & $0.01^{\mathrm{a}}$ & $0.11^{\mathrm{b}}$ & $<0.01$ \\
\hline Entamoebida & 0.00 & 0.00 & 0.00 & NS \\
\hline Unassigned and others & 0.59 & 0.31 & 0.42 & NS \\
\hline
\end{tabular}

Handling protocols: $\mathrm{NH}$ - negative handling $(n=16)$; control - without any handling treatment $(n=8) ; \mathrm{PH}-$ positive handling $(n=8)$; ab - values with different superscripts within each row are significantly different according to Tukey's post-hoc test $(P \leq 0.05)$; NS - indicates lack of a significant difference

growth of negative microorganisms while at the same time significantly reducing beneficial microorganisms. Bailey et al. (2010) demonstrated that exposing mice to a prolonged restraint stressor led to a significant increase in Citrobacter rodentium colonization and suggested that the disruption of the microbiota increases susceptibility to an enteric pathogen.

Although the microbial community structure of PH was clearly separated from the other handling treatments, Ciliophora was the main eukaryote phylum found in the rumen samples. Among the eukaryote community, ciliate protozoa play an important role in rumen digestion of plant cell walls (Ushida and Jouany, 1990). The large difference 
in ciliophoran phyla between handling treatments could be related to the significant change in the rate of plant decomposition. However, as more than half of the PH sheep samples were assigned as unknown or others, it is difficult to come to such a conclusion.

It was found that forage degradability in the treatments with more stress was half that of the treatments with less stress. The most likely explanation for this impaired rumen functionality is that sheep exposed to stress showed different rumen flora from the non-stressed ones, presenting an increase in poor functional microorganisms instead of highly functional organisms and low diversity of rumen organisms. As a result of the fulfilled rumen with the undigested forage may lead to a decrease in voluntary forage intake. This finding is in accordance with other studies, where ruminal acidosis or restraint stress reduced bacterial diversity and altered microflora resulted in indigestion (Mao et al., 2013; Bailey, 2016).

\section{Conclusions}

Handling stress impairs the ability of the rumen to digest hay due to altered rumen microflora. Sheep exposed to stress had more potentially pathogenic bacteria and fewer cellulolytic bacteria such as Firmicutes, as well as their microorganism diversity in the rumen was lower. Within the limitation of this study (only four animals used), it can be carefully concluded that it is important to recognize the potential animal production risks posed by handling stress, which has welfare implications for such farm animals.

\section{Conflict of interest}

The authors declare that there is no conflict of interest.

\section{References}

Bailey M.T., Dowd S.E., Parry N.M., Galley J.D., Schauer D.B., Lyte M., 2010. Stressor exposure disrupts commensal microbial populations in the intestines and leads to increased colonization by Citrobacter rodentium. Infec. Immun. 78, 1509-1519, https:// doi.org/10.1128/IAl.00862-09

Bailey M.T., 2016. Psychological stress, immunity, and the effects on indigenous microflora. Adv. Exp. Med. Biol. 874, 225-246, https://doi.org/10.1007/978-3-319-20215-0_11

Boissy A., Manteuffel G., Jensen M.B., Moe R.O., Spruijt B., Keeling L.J., Bakken M., 2007. Assessment of positive emotions in animals to improve their welfare. Physiol. Behav. 92, 375-397, https:// doi.org/10.1016/j.physbeh.2007.02.003
Breuer K., Hemsworth P.H., Coleman G.J., 2003. The effect of positive or negative handling on the behavioural and physiological responses of nonlactating heifers. Appl. Anim. Behav. Sci. 84, 3-22, https://doi.org/10.1016/S0168-1591(03)00146-1

Broom D.M., Fraser A.F. (Editors), 2015. Domestic Animal Behaviour and Welfare. CABI International. Wallingford (UK), https://doi. org/10.1079/9781780645391.0000

Caporaso J.G., Kuczynski J., Stombaugh J. et al., 2010. QIIME allows analysis of high-throughput community sequencing data. Nat. Methods 7, 335-336, https://doi.org/10.1038/nmeth.f.303

Chang D.H., Rhee M.S., Jeong H., Kim S., Kim B.C., 2015. Draft genome sequence of Acinetobacter sp. HR7, isolated from Hanwoo, Korean Native Cattle. Genome Announcements 3, e01358-14, https://doi.org/10.1128/genomeA.01358-14

Charlton G.L., Rutter S.M., East M., Sinclair L.A., 2011. Effects of providing total mixed rations indoors and on pasture on the behavior of lactating dairy cattle and their preference to be indoors or on pasture. J. Dairy Sci. 94, 3875-3884, https://doi. org/10.3168/jds.2011-4172

Edgar R.C., 2010 Search and clustering orders of magnitude faster than BLAST. Bioinformatics 26, 2460-2461, https://doi. org/10.1093/bioinformatics/btq461

Grandin T. (Editor), 2007. Livestock Handling and Transport. CABI International. Wallingford (UK), https://doi. org/10.1079/9781845932190.0000

Kadzere C.T., Murphy M.R., Silanikove N., Maltz E., 2002. Heat stress in lactating dairy cows: a review. Livest. Prod. Sci. 77, 59-91, https://doi.org/10.1016/S0301-6226(01)00330-X

Khan M.M.H., Chaudhry A.S., 2010. Chemical composition of selected forages and spices and the effect of these spices on in vitro rumen degradability of some forages. Asian-Australas. J. Anim. Sci. 23, 889-900, https://doi.org/10.5713/ajas.2010.90442

Lenz H.J., Raedler A., Greten H., Vale W.W., Rivier J.E., 1988. Stressinduced gastrointestinal secretory and motor responses in rats are mediated by endogenous corticotropin-releasing factor. Gastroenterology 95, 1510-1517, https://doi.org/10.1016/ S0016-5085(88)80070-2

Lindahl C., Pinzke S., Herlin A., Keeling L.J., 2016. Human-animal interactions and safety during dairy cattle handling - Comparing moving cows to milking and hoof trimming. J. Dairy Sci. 99, 2131-2141, https://doi.org/10.3168/jds.2014-9210

Mao S.Y., Zhang R.Y., Wang D.S., Zhu W.Y., 2013. Impact of subacute ruminal acidosis (SARA) adaptation on rumen microbiota in dairy cattle using pyrosequencing. Anaerobe $24,12-19$, https://doi.org/10.1016/j.anaerobe.2013.08.003

McDougall E.I., 1948. Studies on ruminant saliva. 1. The composition and output of sheep's saliva. Biochem. J. 43, 99-109, https:// doi.org/10.1042/bj0430099

Murakami M., Lam S.K., Inada M., Miyake T., 1985. Pathophysiology and pathogenesis of acute gastric mucosal lesions after hypothermic restraint stress in rats. Gastroenterology 88, 660-665, https://doi.org/10.1016/0016-5085(85)90133-7

Russell J.B., Muck R.E., Weimer P.J., 2009. Quantitative analysis of cellulose degradation and growth of cellulolytic bacteria in the rumen. FEMS Microbiol. Ecol. 67, 183-197, https://doi. org/10.1111/j.1574-6941.2008.00633.x

Sasaki H., Takahashi T., Kayaba T., 2000. Effects of the different ratios of concentrate and roughage on the concentration and composition of long chain fatty acids of rumen microorganisms and microorganisms free-liquor in sheep. Anim. Sci. J. 71, 26-38 
Suyama Y., Matsuki Y., 2015. MIG-seq: an effective PCR-based method for genome-wide single-nucleotide polymorphism genotyping using the next-generation sequencing platform. Sci. Rep. 5, 16963, https://doi.org/10.1038/srep16963

Ushida K., Jouany J.P., 1990. Effect of defaunation on fibre digestion in sheep given two isonitrogenous diets. Anim. Feed Sci. Technol. 29, 153-158, https://doi.org/10.1016/03778401(90)90101-D
Von Keyserlingk M.A.G., Rushen J., de Passillé A.M., Weary D.M., 2009. Invited review: The welfare of dairy cattle - Key concepts and the role of science. J. Dairy Sci. 92, 4101-4111, https://doi.org/10.3168/jds.2009-2326

Yardimci M., Sahin E.H., Cetingul I.S., Bayram I., Aslan R., Sengor E., 2013. Stress responses to comparative handling procedures in sheep. Animal 7, 143-150, https://doi.org/10.1017/ S1751731112001449 